\title{
Disconnectivity and Relative Positions in Simultaneous Embeddings ${ }^{\star}$
}

\author{
Thomas Bläsius and Ignaz Rutter \\ Karlsruhe Institute of Technology (KIT) \\ firstname.lastname@kit.edu
}

\begin{abstract}
For two planar graph $G^{(1)}=\left(V^{(1)}, E^{(1)}\right)$ and $G^{(2)}=\left(V^{(2)}, E^{(2)}\right)$ sharing a common subgraph $G=G^{(1)} \cap G^{(2)}$ the problem Simultaneous Embedding with Fixed Edges (SEFE) asks whether they admit planar drawings such that the common graph is drawn the same. Previous algorithms only work for cases where $G$ is connected, and hence do not need to handle relative positions of connected components. We consider the problem where $G, G^{(\mathbb{1}}$ and $G^{(2)}$ are not necessarily connected.

First, we show that a general instance of SEFE can be reduced in linear time to an equivalent instance where $V^{(1)}=V^{(2)}$ and $G^{(1)}$ and $G^{(2)}$ are connected. Second, for the case where $G$ consists of disjoint cycles, we introduce the $C C$-tree which represents all embeddings of $G$ that extend to planar embeddings of $G^{\mathbb{1}}$. We show that CC-trees can be computed in linear time, and that their intersection is again a CC-tree. This yields a linear-time algorithm for SEFE if all $k$ input graphs (possibly $k>2$ ) pairwise share the same set of disjoint cycles. These results, including the CC-tree, extend to the case where $G$ consists of arbitrary connected components, each with a fixed embedding. Then the running time is $O\left(n^{2}\right)$.
\end{abstract}

\section{Introduction}

To enable a human reader to compare different relational datasets on a common set of objects it is important to visualize the corresponding graphs in such a way that the common parts of the different datasets are drawn as similarly as possible. This leads to the fundamental theoretical problem SEFE, asking for two graphs $G^{(1)}=\left(V^{(1)}, E^{(1)}\right)$ and $G^{(2)}=\left(V^{(2)}, E^{(2)}\right)$ with the common graph $G=(V, E)=\left(V^{(1)} \cap V^{(2)}, E^{(1)} \cap E^{(2)}\right)$, whether there are planar drawings of $G^{(1)}$ and $G^{(2)}$ such that the common graph $G$ is drawn the same in both.

The problem SEFE and its variants have been studied extensively in the past years; see [3] for a survey. As there are planar graphs that cannot be embedded simultaneously, the question of deciding whether given graphs admit a SEFE is of high interest. Gassner et al. 8] show that it is $\mathcal{N} \mathcal{P}$-complete to decide SEFE for three or more graphs. For two graphs the complexity status is open. However, there are several approaches yielding efficient algorithms for special cases. Angelini et al. [1] solve SEFE if one of the graphs has a fixed embedding.

\footnotetext{
* Partly done within GRADR - EUROGIGA project no. 10-EuroGIGA-OP-003. 
Fowler at al. show how to solve SEFE efficiently if $G^{(1)}$ and $G$ have at most two and one cycles, respectively [7]. Haeupler et al. solve SEFE in linear time for the case that the common graph is biconnected [10]. Angelini et al. obtain the same result with a completely different approach [2]. The currently least restrictive result by Bläsius and Rutter [4] shows that SEFE can be solved in polynomial time for the case that $G^{(1)}$ and $G^{(2)}$ are biconnected and $G$ is connected.

Jünger and Schulz [11] show that two planar graphs $G^{(1)}$ and $G^{(2)}$ admit a SEFE if and only if they admit planar embeddings that coincide on $G$. All previous testing algorithms assume that $G$ is connected, implying that it is sufficient to enforce the common edges incident to each vertex to have the same circular ordering in both embeddings. Especially in the result by Bläsius and Rutter [4] this is heavily used, as they explicitly consider only orders of edges around vertices using PQ-trees. However, if the common graph is not required to be connected, we additionally have to care about the relative positions of connected components to one another, which introduces an additional difficulty.

Contribution and Outline. We consider disconnectivity in SEFE problems. We give a linear-time algorithm that, for each instance $G^{(1)}$ and $G^{(2)}$ of SEFE, computes an equivalent instance $G^{\mathbb{1}^{\prime}}, G^{()^{\prime}}$ with $G^{(1)} \subseteq$ $G^{1^{\prime}}$ and $G^{(2)} \subseteq G^{()^{\prime}}$ such that all vertices are common and both graphs are connected (Section 2). Note that it is generally not possible to extend $G^{(1)}$ and $G^{(2)}$ to an equivalent instance such that $G$ is connected; see Fig. 1 . In Section 3 we tackle the problem SEFE where the common graph $G$ is disconnected, assuming that the circular order of edges around vertices in $G$ is already fixed and the task is to find compatible relative positions of the connected components of $G$, such that they can be extended to planar embeddings of $G_{1}$ and $G_{2}$. Note that this contrasts previous work, where $G$ is generally assumed to be connected and hence relative positions can be neglected. Initially, we consider the special case where $G$ consists of

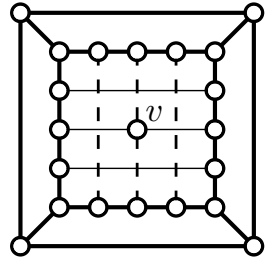

Fig. 1. Bold edges belong to both graphs, the dashed and thin edges are exclusive a set $\mathcal{C}$ of disjoint cycles. We present a novel data structure, the $C C$-tree, that for a planar graph $G$ containing $\mathcal{C}$ represents all planar embeddings of $\mathcal{C}$ that are induced by a planar embedding of $G$. We show that the intersection of two CC-trees is again a CC-tree. CC-trees can be computed and intersected in linear time; this immediately gives a linear-time algorithm for SEFE of graphs $G^{(}, \ldots, G^{\circledR}$ whose pairwise intersection is $\mathcal{C}$. In Section 4 , we extend this to the case where the common graph may contain arbitrary connected components, each with a prescribed planar embedding; in this case the running-time is $O\left(n^{2}\right)$.

\section{Connecting Disconnected Graphs}

Let $G^{(1)}=\left(V, E^{(1)}\right)$ and $G^{(2)}=\left(V, E^{(2)}\right)$ be two planar graphs with common graph $G=(V, E)$ with $E=E^{(1)} \cap E^{(2)}$. We show that the problem SEFE can be reduced 
to the case where $G^{(1)}$ and $G^{(2)}$ are connected. We can assume that $G^{(1)} \cup G^{(2)}$ is connected, otherwise the connected components can be handled independently. We first ensure that $G^{(1)}$ is connected without increasing the number of connected components in $G^{(2)}$. Afterwards we apply the same steps to $G^{(2)}$.

Let $k^{(1)}$ and $k^{(2)}$ denote the number of connected components of $G^{(1)}$ and $G^{(2)}$, respectively, and assume $k^{(1)}>1$. Since $G^{(1)} \cup G^{(2)}$ is connected there exists an edge $e^{(2)}=\left\{v_{1}, v_{2}\right\} \in E^{(2)}$ connecting vertices from different connected components $H_{1}^{(1)}$ and $H_{2}^{(1)}$ in $G^{(1)}$. We construct the augmented instance $\left(G_{+}^{(1)}, G_{+}^{(2)}\right)$ with respect to the edge $e^{(2)}$ by adding a new vertex $v_{12}$ and edges $e=\left\{v_{1}, v_{12}\right\} \in E$ and $e^{(1)}=\left\{v_{12}, v_{2}\right\} \in E^{(1)}$. Note that $G_{+}^{(1)}$ has $k^{(1)}-1$ connected components, and the number of connected components in $G^{(2)}$ does not change.

Lemma 1. The SEFE instances $\left(G^{(1)}, G^{(2)}\right)$ and $\left(G_{+}^{(1)}, G_{+}^{(2)}\right)$ are equivalent.

Proof. If $\left(G_{+}^{(1)}, G_{+}^{(2)}\right)$ admits a SEFE, then obviously $\left(G^{(1)}, G^{(2)}\right)$ does. For the converse assume that $\left(G^{(1)}, G^{(2)}\right)$ has a $\operatorname{SEFE}\left(\mathcal{E}^{(1)}, \mathcal{E}^{(2)}\right)$ inducing the embedding $\mathcal{E}$ on $G$. We construct an embedding $\mathcal{E}^{\prime(1)}$ such that (i) $\left(\mathcal{E}^{\prime(1)}, \mathcal{E}^{(2)}\right)$ is a SEFE, and (ii) the vertices $v_{1}$ and $v_{2}$ lie on a common face in $\mathcal{E}^{\prime(1)}$. Then we can add the vertex $v_{12}$ together with the two edges $e$ and $e^{(1)}$, yielding a SEFE of $\left(G_{+}^{(1)}, G_{+}^{(2)}\right)$.

Consider a face $f$ of $\mathcal{E}$. For $i=1,2$, the embedding $\mathcal{E}^{(1)}$ of $G^{(1)}$ splits $f$ into a set of faces $\mathcal{F}^{\oplus}(f)=\left\{f_{1}^{\oplus}, \ldots, f_{k}^{\oplus}\right\}$. We say that a face $f^{\oplus} \in \mathcal{F}^{\oplus}(f)$ is contained in $f$. Note that every face of $\mathcal{E}^{(1)}$ is contained in exactly one face of $\mathcal{E}$.

The edge $e^{(2)}=\left\{v_{1}, v_{2}\right\}$ is incident to two faces $f_{1}^{(2)}$ and $f_{2}^{(2)}$ of $\mathcal{E}^{(2)}$. Since $e^{(2)}$ belongs exclusively to $G^{(2)}$, they are both contained in the same face $f$ of $\mathcal{E}$; without loss of generality $f$ is the outer face. In $\mathcal{E}^{(1)}$, the face $f$ may be subdivided into several faces. However, we can find faces $f_{1}^{(1)}$ and $f_{2}^{(1)}$ of $\mathcal{E}^{(1)}$, both contained in $f$, such that $v_{1}$ and $v_{2}$ are incident to $f_{1}^{(1)}$ and $f_{2}^{(1)}$, respectively. Since $v_{1}$ and $v_{2}$ belong to distinct components of $G^{(\mathbb{1}}$, there exists a closed curve $C$ in the plane separating $G^{(\mathbb{1}}$ into two subgraphs $G_{1}^{(\mathbb{1})}$ and $G_{2}^{(1)}$ lying to the left and to the right of $C$, and containing $v_{1}$ and $v_{2}$, respectively. Let $\mathcal{E}_{1}^{(1)}$ and $\mathcal{E}_{2}^{(1)}$ denote the planar embeddings of $G_{1}^{(1)}$ and $G_{2}^{(1)}$ induced by $\mathcal{E}^{(1)}$. We now choose $f_{1}^{(1)}$ and $f_{2}^{(1)}$ as the outer face of $\mathcal{E}_{1}^{(1)}$ and $\mathcal{E}_{2}^{(1)}$, respectively. Note that this uniquely induces a planar embedding $\mathcal{E}^{\prime(1)}$ of $G$ where $v_{1}$ and $v_{2}$ are on the outer face (property

(ii)). Moreover, since $f_{1}^{(1)}$ and $f_{2}^{(1)}$ are both contained in the outer face $f$ of $\mathcal{E}$, the embedding of $G$ is preserved. Hence, $\left(\mathcal{E}^{(1)}, \mathcal{E}^{(2)}\right)$ is still a SEFE.

Iteratively applying the operation to suitably chosen edges yields the following.

Theorem 1. There is a linear-time algorithm that computes for any instance of SEFE an equivalent instance such that both graphs are connected.

\section{Disjoint Cycles}

In this section, we consider the problem SEFE for the case that the common graph consists of a set of disjoint cycles. Due to Theorem 1 we may assume that both graphs are connected. In Section 3.1 we show how to solve this special case 
of SEFE in polynomial time. In Section 3.2 we introduce a tree-like data structure, the $C C$-tree, representing all planar embeddings of a set of cycles contained in a single graph that can be induced by an embedding of the whole graph. We additionally show that the intersection of the set of embeddings represented by two CC-trees can again be represented by a CC-tree, yielding a solution for SEFE even for the case of more than two graphs if all graphs have the same intersection consisting of a set of disjoint cycles. We further show how to compute and intersect CC-trees in linear time. Due to space constraints, some proofs are omitted. Detailed proofs can be found in the full version of this paper, which is in the appendix. Before we start, we fix some definitions.

Embeddings of Disjoint Cycles. Let $C_{1}, \ldots, C_{k}$ be a set of disjoint simple cycles. We consider embeddings of these cycles on the sphere. Since a single cycle has a unique embedding on the sphere only their relative positions are of interest. To be able to use the terms "left" and "right", we direct the cycles arbitrarily. We denote the relative position of a cycle $C_{j}$ with respect to a cycle $C_{i}$ by $\operatorname{pos}_{C_{i}}\left(C_{j}\right)$. More precisely, we have $\operatorname{pos}_{C_{i}}\left(C_{j}\right)=$ "left" and $\operatorname{pos}_{C_{i}}\left(C_{j}\right)=$ "right", if $C_{j}$ lies on the left and right side of $C_{i}$, respectively. We call an assignment of a value "left" or "right" to each of these relative positions a semi-embedding of the cycles $C_{1}, \ldots, C_{k}$. Note that the embeddings are a strict subset of the semi-embeddings, i.e., not every semi-embedding yields an embedding of the cycles. We extend our notation to relative positions of arbitrary subgraphs. For example the relative position of a single vertex $v$ with respect to a cycle $C$ is denoted by $\operatorname{pos}_{C}(v)$.

$S P Q R$-Tree. As connectivity plays an important role in this work we fix some basic definitions in the following. A graph is connected if there exists a path between any pair of vertices. A separating $k$-set is a set of $k$ vertices whose removal disconnects the graph. Separating 1-sets and 2-sets are cutvertices and separation pairs, respectively. A connected graph is biconnected if it does not have a cut vertex and triconnected if it does not have a separation pair. The maximal biconnected components of a graph are called blocks. The cut components with respect to a separating $k$-set $S$ are the maximal subgraphs that are not disconnected by removing $S$.

We use the $S P Q R$-tree introduced by Di Battista and Tamassia [5]6] to represent all planar embeddings of a biconnected planar graph $G$. The SPQR-tree $\mathcal{T}$ of $G$ is a decomposition of $G$ into triconnected components along its split pairs, where a split pair is either a separating pair or a pair of adjacent vertices. We define the SPQR-tree to be unrooted, representing embeddings on the sphere, that is planar embeddings without a designated outer face.

Let $\{s, t\}$ be a split pair and let $H_{1}$ and $H_{2}$ be two subgraphs of $G$ such that $H_{1} \cup H_{2}=G$ and $H_{1} \cap H_{2}=\{s, t\}$. Consider the tree consisting of two nodes $\mu_{1}$ and $\mu_{2}$ associated with the graphs $H_{1}+\{s, t\}$ and $H_{2}+\{s, t\}$, respectively. These graphs are called skeletons of the nodes $\mu_{i}$, denoted by $\operatorname{skel}\left(\mu_{i}\right)$, and the special edge $\{s, t\}$ is a virtual edge. The edge connecting the nodes $\mu_{1}$ and $\mu_{2}$ associates the virtual edges in $\operatorname{skel}\left(\mu_{1}\right)$ and $\operatorname{skel}\left(\mu_{2}\right)$ with each other. The expansion graph $\exp (\{s, t\})$ of a virtual edge $\{s, t\}$ is the subgraph of $G$ it represents, 

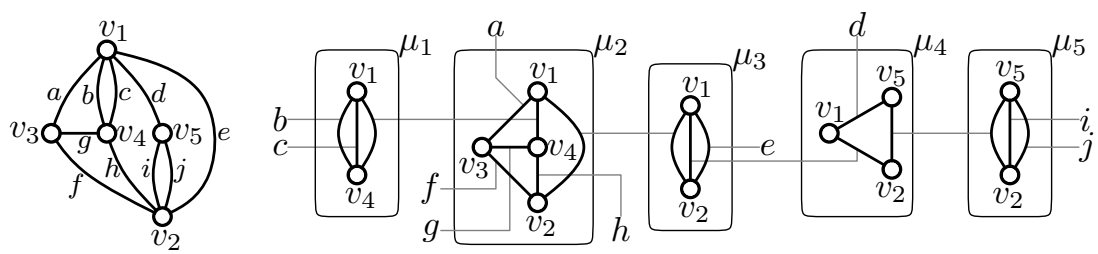

Fig. 2. The SPQR-tree of a biconnected planar graph. The nodes $\mu_{1}, \mu_{3}$ and $\mu_{5}$ are $\mathrm{P}$-nodes, $\mu_{2}$ is an R-node and $\mu_{4}$ is an S-node; Q-nodes are not shown.

that is in $\operatorname{skel}\left(\mu_{1}\right)$ the expansion graph of $\{s, t\}$ is $H_{2}$ and the expansion graph of $\{s, t\}$ in $\operatorname{skel}\left(\mu_{2}\right)$ is $H_{1}$. Now a combinatorial embedding of $G$ uniquely induces a combinatorial embedding of $\operatorname{skel}\left(\mu_{1}\right)$ and $\operatorname{skel}\left(\mu_{2}\right)$ and vice versa.

Applying this kind of decomposition systematically yields the SPQR-tree; see Fig. 2. The skeletons of the internal nodes of the SPQR-tree are either a cycle (S-node), a bunch of parallel edges (P-node) or a triconnected planar graph (R-node). The leaves are Q-nodes, and their skeleton consists of two vertices connected by a virtual and a normal edge. Thus, the only possible embedding choices are flipping skeletons of R-nodes (triconnected graphs have a unique embedding up to a flip [12]) and ordering the edges in skeletons of P-nodes. The SPQR-tree can be computed in linear time [9].

\subsection{A Polynomial-Time Algorithm}

Let $\left(G^{(1)}, G^{(2)}\right)$ be an instance of SEFE with common graph $G$ consisting of pairwise disjoint simple cycles $C_{1}, \ldots, C_{k}$. We first assume that $G^{(1)}$ and $G^{(2)}$ are biconnected and show later how to remove this restriction. Our approach is to formulate constraints on the relative positions of the cycles to one another ensuring that $G^{(1)}$ and $G^{(2)}$ induce the same semi-embedding of the common graph $G$. We show implicitly that the resulting semi-embedding is really an embedding by showing that the graphs $G^{(1)}$ and $G^{(2)}$ have planar embeddings inducing this semi-embedding. Note that this only works for the case that $G^{(1)}$ and $G^{(2)}$ are connected. Thus, our approach crucially relies on the result from Section 2.

Biconnected Graphs. Before considering two graphs, we determine for a single graph the possible embeddings it may induce on a set of disjoint cycles contained in it. Let $G=(V, E)$ be a biconnected graph with SPQR-tree $\mathcal{T}$, let $C$ be a simple directed cycle in $G$ and let $\mu$ be a node in $\mathcal{T}$. Obviously, $C$ is either completely contained in the expansion graph of a single virtual edge of $\mu$ or $C$ induces a simple directed cycle of virtual edges in $\operatorname{skel}(\mu)$. We say that $C$ is contracted and a cycle in $\operatorname{skel}(\mu)$ in the former and latter case, respectively. Consider the case where $C$ is a cycle in $\operatorname{skel}(\mu)$ and let $\kappa$ denote this cycle. By fixing the embedding of $\operatorname{skel}(\mu)$ the virtual edges in $\operatorname{skel}(\mu)$ not contained in $\kappa$ split into two groups, some lie to the left and some to the right of $\kappa$. Obviously, a vertex $v \in V \backslash V(C)$ in the expansion graph of a virtual edge that lies to the left (to the right) of $\kappa$ 
lies to the left (to the right) of $C$ in $G$, no matter which embedding is chosen for the skeletons of other nodes. In other words, the value of $\operatorname{pos}_{C}(v)$ is completely determined by this single node $\mu$. We show that for every vertex $v \in V \backslash V(C)$ there is a node $\mu$ containing $C$ as a cycle such that the virtual edge in $\operatorname{skel}(\mu)$ containing $v$ in its expansion graph is not contained in the cycle $\kappa$ induced by $C$. Hence a node $\mu$ in $\mathcal{T}$ determining $\operatorname{pos}_{C}(v)$ always exists. Extending this to a pair of cycles yields the following lemma.

Lemma 2. Let $G$ be a biconnected planar graph with $S P Q R$-tree $\mathcal{T}$ and let $C_{1}$ and $C_{2}$ be two disjoint simple cycles in $G$. There is exactly one node $\mu$ in $\mathcal{T}$ determining $\operatorname{pos}_{C_{1}}\left(C_{2}\right)$. Moreover, $C_{1}$ is a cycle $\kappa_{1}$ in $\operatorname{skel}(\mu)$ and $C_{2}$ is either a cycle or contracted in a virtual edge not contained in $\kappa_{1}$.

Now consider a set of pairwise disjoint cycles $\mathcal{C}=\left\{C_{1}, \ldots, C_{k}\right\}$ in $G$. Obviously $\mathrm{S}$ - and Q-nodes do not determine any relative positions. Let $\mu$ be an arbitrary $\mathrm{P}$ - or R-node in the SPQR-tree $\mathcal{T}$ and let $C \in \mathcal{C}$ be a cycle that occurs as a cycle $\kappa$ in $\operatorname{skel}(\mu)$.

Let $\mu$ be a $\mathbf{P}$-node with vertices $s$ and $t$ and parallel virtual edges $\varepsilon_{1}, \ldots, \varepsilon_{\ell}$ between them. Then $\kappa$ consists of two parallel virtual edges, without loss of generality $\varepsilon_{1}$ and $\varepsilon_{2}$. By disjointness no other cycle $C^{\prime} \in \mathcal{C}$ is a cycle in skel $(\mu)$ as $C^{\prime}$ would also contain $s$ and $t$. Thus, every other cycle $C^{\prime}$ is contracted in $\operatorname{skel}(\mu)$, and belongs to one of the virtual edges $\varepsilon_{1}, \ldots, \varepsilon_{\ell}$. If it belongs to $\varepsilon_{1}$ or $\varepsilon_{2}$, which are contained in $\kappa$, then $\operatorname{pos}_{C}\left(C^{\prime}\right)$ is not determined by $\mu$. Otherwise, $C^{\prime}$ belongs to one of the virtual edges $\varepsilon_{3}, \ldots, \varepsilon_{\ell}$, and $\operatorname{pos}_{C}\left(C^{\prime}\right)$ is determined by the relative position of this virtual edge with respect to the cycle $\kappa$. This relative position can be chosen for every virtual edge $\varepsilon_{3}, \ldots, \varepsilon_{\ell}$ arbitrarily and independently. However, if two cycles $C_{i}$ and $C_{j}$ belong to the same virtual edge $\varepsilon \in\left\{\varepsilon_{3}, \ldots, \varepsilon_{\ell}\right\}$, their relative position with respect to $C$ is the same, that is $\operatorname{pos}_{C}\left(C_{i}\right)=\operatorname{pos}_{C}\left(C_{j}\right)$, for every embedding of $G$.

Let $\mu$ be an $\mathbf{R}$-node. For the moment, we consider the embedding of $\operatorname{skel}(\mu)$ to be fixed. The relative position $\operatorname{pos}_{C}\left(C^{\prime}\right)$ of a cycle $C^{\prime} \neq C$ is determined by $\mu$ if and only if $C^{\prime}$ is a cycle in $\operatorname{skel}(\mu)$ or if it is contracted and belongs to a virtual edge not contained in $\kappa$. Since we consider only one of the two embeddings of $\operatorname{skel}(\mu)$ at the moment, $\operatorname{pos}_{C}\left(C^{\prime}\right)$ is fixed to one of the two values "left" or "right" in this case. The same can be done for all other cycles that are cycles in $\operatorname{skel}(\mu)$ yielding a fixed value for all relative positions that are determined by $\mu$. Finally, we have a partition of all positions determined by $\mu$ into the set of positions $\mathcal{P}_{1}=\left\{\operatorname{pos}_{C_{a(1)}}\left(C_{b(1)}\right), \ldots, \operatorname{pos}_{C_{a(r)}}\left(C_{b(r)}\right)\right\}$ all having the value "left" and the set of positions $\mathcal{P}_{2}=\left\{\operatorname{pos}_{C_{c(1)}}\left(C_{d(1)}\right), \ldots, \operatorname{pos}_{C_{c(s)}}\left(C_{d(s)}\right)\right\}$ having the value "right". Now if the embedding of $\operatorname{skel}(\mu)$ is not fixed anymore, we have only the possibility to flip it. By flipping, all the positions in $\mathcal{P}_{1}$ change to "right" and all positions in $\mathcal{P}_{2}$ change to "left". Hence, we obtain that the equation $\operatorname{pos}_{C_{a(1)}}\left(C_{b(1)}\right)=\cdots=\operatorname{pos}_{C_{a(r)}}\left(C_{b(r)}\right) \neq \operatorname{pos}_{C_{c(1)}}\left(C_{d(1)}\right)=\cdots=\operatorname{pos}_{C_{c(s)}}\left(C_{d(s)}\right)$ is satisfied for every embedding of $C_{1}, \ldots, C_{k}$ induced by an embedding of $G$.

To sum up, we obtain a set of (in)equalities relating the relative positions of cycles to one another. We call these constraints the PR-node constraints with 
respect to the biconnected graph $G$. Obviously the PR-node constraints are necessary in the sense that every embedding of $G$ induces an embedding of the cycles $C_{1}, \ldots, C_{k}$ satisfying these constraints. In the full version of the paper we show that, conversely, given a semi-embedding $\mathcal{E}_{\mathcal{C}}$ satisfying the PR-node constraints, one can choose suitable embeddings for all skeletons of the SPQRtree yielding a planar embedding $\mathcal{E}$ of $G$ inducing $\mathcal{E}_{\mathcal{C}}$. Note that this implicitly shows that $\mathcal{E}_{\mathcal{C}}$ is not only a semi-embedding but also an embedding of $\mathcal{C}$.

Lemma 3. Let $G$ be a biconnected planar graph containing the disjoint cycles $C_{1}, \ldots, C_{k}$. Let further $\mathcal{E}_{\mathcal{C}}$ be a semi-embedding of these cycles. There is an embedding $\mathcal{E}$ of $G$ inducing $\mathcal{E}_{\mathcal{C}}$ if and only if $\mathcal{E}_{\mathcal{C}}$ satisfies the PR-node constraints.

Now let $G^{(1)}$ and $G^{(2)}$ be two biconnected planar graphs with the common graph $G$ consisting of pairwise disjoint simple cycles $C_{1}, \ldots, C_{k}$. If we find a semiembedding $\mathcal{E}$ of the cycles that satisfies the PR-node constraints with respect to $G^{(1)}$ and $G^{(2)}$ simultaneously, we can use Lemma 3 to find embeddings $\mathcal{E}^{(1)}$ and $\mathcal{E}^{(2)}$ for $G^{(1)}$ and $G^{(2)}$ both inducing the embedding $\mathcal{E}$ on the common graph $G$. Thus, satisfying the PR-node constraints with respect to both $G^{(1)}$ and $G^{(2)}$ is sufficient to find a SEFE. Conversely, given a pair of embeddings $\mathcal{E}^{(1)}$ and $\mathcal{E}^{(2)}$ inducing the same embedding $\mathcal{E}$ on $G$, this embedding $\mathcal{E}$ needs to satisfy the PR-node constraints with respect to both, $G^{(1)}$ and $G^{(2)}$, which is again due to Lemma 3. Since the PR-node constraints form a set of boolean (in)equalities we can express them as an instance of 2-SAT. As this instance has polynomial size and can easily be computed in polynomial time, we obtain the following theorem.

Theorem 2. SEFE can be solved in $O\left(n^{2}\right)$ time for biconnected graphs whose common graph is a set of disjoint cycles.

Allowing Cutvertices. Next, we generalize our observations to graphs that may contain cutvertices. As before, we consider a single graph $G$ containing a set of disjoint cycles $\mathcal{C}=\left\{C_{1}, \ldots, C_{k}\right\}$ first. Let $C \in \mathcal{C}$ be one of the cycles and let $v$ be a cutvertex contained in the same block $B$ that contains $C$. The cutvertex $v$ splits $G$ into $\ell$ components $H_{1}, \ldots, H_{\ell}$. Assume without loss of generality that $B$ (and with it also $C$ ) is contained in $H_{1}$. We distinguish between the cases that $v$ is contained in $C$ and that it is not.

If $\boldsymbol{v}$ is not contained in $\boldsymbol{C}$, then the relative position $\operatorname{pos}_{C}(v)$ is determined by the embedding of the block $B$ and it follows that all the subgraphs $H_{2}, \ldots, H_{\ell}$ lie on the same side of $C$ as $v$ does. It follows from the biconnected case that $\operatorname{pos}_{C}(v)$ is determined by the embedding of the skeleton of exactly one node $\mu$ in the SPQR-tree of $B$. Obviously, the conditions that all cycles in $H_{2}, \ldots, H_{\ell}$ are on the same side of $C$ as $v$ can be easily added to the PR-node constraints stemming from the node $\mu$; call the resulting constraints the extended PR-node constraints.

If $\boldsymbol{v}$ is contained in $\boldsymbol{C}$, the relative position $\operatorname{pos}_{C}(v)$ does not exist. Assume the embedding of each block is already chosen. Then for each of the subgraphs 
$H \in\left\{H_{2}, \ldots, H_{\ell}\right\}$ the positions $\operatorname{pos}_{C}(H)$ can be chosen arbitrarily and independently. In this case we say for a cycle $C^{\prime}$ in $H$ that its relative position $\operatorname{pos}_{C}\left(C^{\prime}\right)$ is determined by the embedding chosen for the cutvertex $v$. Obviously, in every embedding of $G$ a pair of cycles $C_{i}$ and $C_{j}$ both belonging to the same subgraph $H \in\left\{H_{2}, \ldots, H_{\ell}\right\}$ lie on the same side of $C$ yielding the equation $\operatorname{pos}_{C}\left(C_{i}\right)=\operatorname{pos}_{C}\left(C_{j}\right)$. This equation can be set up for every pair of cycles in each of the subgraphs, yielding the cutvertex constraints with respect to $v$.

Clearly, a semi-embedding $\mathcal{E}_{\mathcal{C}}$ on the cycles $C_{1}, \ldots, C_{k}$ that is induced by an embedding $\mathcal{E}$ of the whole graph always satisfies the extended PR-node and cutvertex constraints. As before, the converse is also true; the proof is omitted.

Lemma 4. Let $G$ be a connected planar graph containing the disjoint cycles $C_{1}, \ldots, C_{k}$. Let further $\mathcal{E}_{\mathcal{C}}$ be a semi-embedding of these cycles. There is an embedding $\mathcal{E}$ of $G$ inducing $\mathcal{E}_{\mathcal{C}}$ if and only if $\mathcal{E}_{\mathcal{C}}$ satisfies the extended PR-node and cutvertex constraints.

This result again directly yields a polynomial-time algorithm to solve SEFE for the case that both graphs $G^{\circledR}$ and $\left.G^{(}\right)$are connected and their common graph $G$ consists of a set of disjoint cycles. Moreover, requiring both graphs to be connected is not really a restriction due to Theorem 1. The extended PR-node and cutvertex constraints can be computed similarly as for Theorem 2, yielding the following theorem.

Theorem 3. SEFE can be solved in $O\left(n^{2}\right)$ time if the common graph consists of disjoint cycles.

\subsection{A Compact Representation of All Simultaneous Embeddings}

In the previous section we showed that SEFE can be solved in polynomial time for the case that the common graph consists of disjoint cycles. In this section we describe a data-structure, the CC-tree, representing all embeddings of a set of disjoint cycles that can be induced by an embedding of a connected graph containing them. Afterwards, we show that the intersection of the sets of embeddings represented by two CC-trees can again be represented by a CC-tree. Moreover, we can show that the CC-tree and the intersection of two CC-trees can be computed in linear time, yielding an optimal linear-time algorithm for SEFE for the case that the common graph consists of disjoint cycles. Note that this algorithm obviously extends to the case where $k$ graphs $G^{\circledR}, \ldots, G^{\circledR}$ are given such that they all intersect in the same common graph $G$ consisting of a set of disjoint cycles.

C-Trees and CC-Trees. Let $\mathcal{C}=\left\{C_{1}, \ldots, C_{k}\right\}$ be a set of disjoint cycles. A cycle-tree (C-tree) $\mathcal{T}_{\mathcal{C}}$ on these cycles is a minimal connected graph containing $\mathcal{C}$. Obviously, every embedding of $\mathcal{T}_{\mathcal{C}}$ induces an embedding of the cycles. We say that two embeddings of $\mathcal{T}_{\mathcal{C}}$ are equivalent if they induce the same embedding of $\mathcal{C}$ and we are only interested in the equivalence classes with respect to this 
equivalence relation. An embedding $\mathcal{E}$ of the cycles in $\mathcal{C}$ is represented by $\mathcal{T}_{\mathcal{C}}$ if it admits an embedding inducing $\mathcal{E}$. Note that contracting each of the cycles $C_{1}, \ldots, C_{k}$ in a C-tree to a single vertex yields a spanning tree on these vertices. In most cases we implicitly assume the cycles to be contracted such that $\mathcal{T}_{\mathcal{C}}$ can be treated like a tree.

The embedding choices that can be made for $\mathcal{T}_{\mathcal{C}}$ are of the following kind. For every edge $e=\left\{C, C^{\prime}\right\}$ we can decide to put all cycles in the subtree attached to $C$ via $e$ either to the left or to the right of $C$. In particular, we can assign a value "left" or "right" to the relative position $\operatorname{pos}_{C}\left(C^{\prime}\right)$. Moreover, by fixing the relative positions $\operatorname{pos}_{C}\left(C^{\prime}\right)$ and $\operatorname{pos}_{C^{\prime}}(C)$ for every pair of cycles $C$ and $C^{\prime}$ that are adjacent in $\mathcal{T}_{\mathcal{C}}$, the embedding represented by $\mathcal{T}_{\mathcal{C}}$ is completely determined. Note that these relative positions can be chosen independently from one another. We call these relative positions crucial relative positions with respect to $\mathcal{T}_{\mathcal{C}}$.

Since the crucial relative positions with respect to a $\mathrm{C}$-tree $\mathcal{T}_{\mathcal{C}}$ are binary variables, we can use (in)equalities between them to further constrain the embeddings represented by $\mathcal{T}_{\mathcal{C}}$. We call a $\mathrm{C}$-tree with such additional constraints on its crucial relative positions a constrained cycle-tree (CC-tree) on the set of cycles $\mathcal{C}$. The CC-tree $\mathcal{T}_{\mathcal{C}}$ represents the embeddings represented by the underlying $\mathrm{C}$-tree whose crucial relative positions satisfy the additional constraints, i.e., there is a bijection between the represented embeddings and the solutions of the 2-SAT instance given by these constraints. We essentially prove two things. First, for every connected graph $G$ containing the cycles $\mathcal{C}$ there exists a CC-tree representing exactly the embeddings of $\mathcal{C}$ that can be induced by embeddings of $G$. Essentially, we have to restrict the extended PR-node and cutvertex constraints to the crucial relative positions of a C-tree compatible with $G$. Second, for a pair of CC-trees $\mathcal{T}_{\mathcal{C}}^{(1)}$ and $\mathcal{T}_{\mathcal{C}}^{(2)}$ on the same set of cycles there exists a CC-tree $\mathcal{T}_{\mathcal{C}}$ representing exactly the embeddings on $\mathcal{C}$ that are represented by $\mathcal{T}_{\mathcal{C}}^{(1)}$ and $\mathcal{T}_{\mathcal{C}}^{(2)}$.

Let $G$ be a connected planar graph containing the disjoint cycles $\mathcal{C}$. We say that a $\mathrm{C}$-tree $\mathcal{T}_{\mathcal{C}}$ on the cycles $\mathcal{C}$ is compatible with $G$ if it is a minor of $G$. Note that $G$ may have many compatible C-trees, in the following we fix one of them and call it the $\mathrm{C}$-tree of $G$. The CC-tree $\mathcal{T}_{\mathcal{C}}$ compatible with $G$ is the C-tree of $G$ together with the restriction of the extended PR-node and cutvertex constraints to the crucial relative positions with respect to $\mathcal{T}_{\mathcal{C}}$.

Theorem 4. Let $G$ be a connected planar graph containing the disjoint cycles $\mathcal{C}$. The $C C$-tree $\mathcal{T}_{\mathcal{C}}$ of $G$ represents exactly the embeddings of $\mathcal{C}$ that can be induced by an embedding of $G$.

Sketch of proof. An embedding $\mathcal{E}$ of $G$ induces an embedding $\mathcal{E}_{\mathcal{C}}$ of the cycles $C_{1}, \ldots, C_{k}$. Since the C-tree $\mathcal{T}_{\mathcal{C}}$ is a minor of $G$, it represents $\mathcal{E}_{\mathcal{C}}$. Moreover, by Lemma $4, \mathcal{E}_{c}$ satisfies the extended PR-node and cutvertex constraints. In particular these constraints hold for all crucial relative positions, and hence $\mathcal{E}_{\mathcal{C}}$ is represented by the $\mathrm{CC}$-tree $\mathcal{T}_{\mathcal{C}}$. The converse is not that simple. However, it can be shown that if the extended PR-node and cutvertex constraints are satisfied for all crucial relative positions, then they also hold for all relative positions. Then an embedding can be constructed as in Lemma 4. 
Intersecting CC-Trees. Next, we consider two CC-trees $\mathcal{T}_{\mathcal{C}}^{\mathbb{1}}$ and $\mathcal{T}_{\mathcal{C}}^{(2)}$ on the same set of cycles $\mathcal{C}$. We show that the set of embeddings that are represented by both $\mathcal{T}_{\mathcal{C}}^{\circledR}$ and $\mathcal{T}_{\mathcal{C}}^{(2)}$ can again be represented by a single CC-tree. We show this by constructing a new CC-tree, which we call the intersection of $\mathcal{T}_{\mathcal{C}}^{\circledR}$ and $\mathcal{T}_{\mathcal{C}}^{(2)}$, showing afterwards that this $\mathrm{CC}$-tree has the desired property. The intersection $\mathcal{T}_{\mathcal{C}}$ is a copy of $\mathcal{T}_{\mathcal{C}}^{\circledR}$ with some additional constraints given by the second CC-tree $\mathcal{T}_{\mathcal{C}}^{2}$. We essentially have to formulate two types of constraints. First, constraints stemming from the structure of the underlying C-tree of $\mathcal{T}_{\mathcal{C}}^{(2)}$. Second, the constraints given by the (in)equalities on the relative positions that are crucial with respect to $\mathcal{T}_{\mathcal{C}}^{(2)}$. We show that both kinds of constraints can be formulated as (in)equalities on the relative positions that are crucial with respect to $\mathcal{T}_{\mathcal{C}}^{\mathbb{D}}$.

Let $C_{1}$ and $C_{2}$ be two cycles joined by an edge in $\mathcal{T}_{\mathcal{C}}^{(2)}$. Obviously, $C_{1}$ and $C_{2}$ are contained in the boundary of a common face in every embedding $\mathcal{E}^{\circledR}$ represented by $\mathcal{T}_{\mathcal{C}}^{(2)}$. It is easy to formulate constraints on the relative positions that are crucial with respect to $\mathcal{T}_{\mathcal{C}}^{\circledR}$ such that $C_{1}$ and $C_{2}$ also share a common face in every embedding represented by $\mathcal{T}_{\mathcal{C}}^{\circledR}$. Consider the path $\pi$ from $C_{1}$ to $C_{2}$ in $\mathcal{T}_{\mathcal{C}}^{\circledR}$. For every three cycles $C, C^{\prime}$ and $C^{\prime \prime}$ appearing consecutively on $\pi$ it is necessary that $\operatorname{pos}_{C^{\prime}}(C)=\operatorname{pos}_{C^{\prime}}\left(C^{\prime \prime}\right)$ holds, otherwise $C_{1}$ and $C_{2}$ would be separated by $C^{\prime}$. Conversely, if this equation holds for every triple of consecutive cycles on $\pi$, then $C_{1}$ and $C_{2}$ always lie on a common face. We call the resulting equations the common-face constraints. Note that all relative positions involved in such constraints are crucial with respect to $\mathcal{T}_{\mathcal{C}}^{\circledR}$. Moreover, it can be shown that the common-face constraints have size $O(n)$. To see this, root $\mathcal{T}_{\mathcal{C}}^{\circledR}$ at an arbitrary node. For a node $C$ with parent $C^{\prime}$ there are at most $O(\operatorname{deg}(C))$ common-face constraints involving $\operatorname{pos}_{C}\left(C^{\prime}\right)$. Hence the number of these constraints is $O(n)$. Any other common-face constraint at $C$ is of the form $\operatorname{pos}_{C}\left(C_{1}\right)=\operatorname{pos}_{C}\left(C_{2}\right)$, where $C_{1}$ and $C_{2}$ are children of $C$. Each such constraint can be attributed to an edge $\left\{C_{1}^{\prime}, C_{2}^{\prime}\right\}$ in $\mathcal{T}_{\mathcal{C}}^{(2)}$ such that $C$ is the lowest common ancestor of $C_{1}^{\prime}$ and $C_{2}^{\prime}$ in $\mathcal{T}_{\mathcal{C}}^{(\mathbb{1}}$. The claim follows since no edge in $\mathcal{T}_{\mathcal{C}}^{(2)}$ is responsible for more than one of these common-face constraints.

To formulate the constraints given on the crucial relative positions of $\mathcal{T}_{\mathcal{C}}^{(2)}$, we essentially find, for each of these crucial relative positions $\operatorname{pos}_{C_{1}}\left(C_{2}\right)$, a relative position $\operatorname{pos}_{C_{1}}\left(C_{2}^{\prime}\right)$ that is crucial with respect to $\mathcal{T}_{\mathcal{C}}^{\circledR}$ such that $\operatorname{pos}_{C_{1}}\left(C_{2}\right)$ is determined by fixing $\operatorname{pos}_{C_{1}}\left(C_{2}^{\prime}\right)$ in $\mathcal{T}_{\mathcal{C}}^{\circledR}$. More precisely, for every relative position $\operatorname{pos}_{C_{1}}\left(C_{2}\right)$ that is crucial with respect to $\mathcal{T}_{\mathcal{C}}^{\text {(2) }}$ we define its representative in $\mathcal{T}_{\mathcal{C}}^{\mathbb{1}}$ to be the crucial relative position $\operatorname{pos}_{C_{1}}\left(C_{2}^{\prime}\right)$ where $C_{2}^{\prime}$ is the first cycle in $\mathcal{T}_{\mathcal{C}}^{\circledR}$ on the path from $C_{1}$ to $C_{2}$. We obtain the crucial-position constraints on the crucial relative positions of $\mathcal{T}_{\mathcal{C}}^{(1)}$ by replacing every relative position in the constraints given for $\mathcal{T}_{\mathcal{C}}^{(2)}$ by its representative. The resulting set of (in)equalities on the crucial relative positions of $\mathcal{T}_{\mathcal{C}}^{\circledR}$ has size $O(n)$ and is obviously necessary.

We can now formally define the intersection $\mathcal{T}_{\mathcal{C}}$ of two CC-trees $\mathcal{T}_{\mathcal{C}}^{(1)}$ and $\mathcal{T}_{\mathcal{C}}^{(2)}$ to be $\mathcal{T}_{\mathcal{C}}^{\mathbb{1}}$ with the common-face and crucial-position constraints additionally restricting its crucial relative positions. We obtain the following theorem, justifying the name "intersection". 
Theorem 5. The intersection of two CC-trees represents exactly the embeddings that are represented by both CC-trees.

Sketch of proof. First observe that we only added necessary conditions for representability in $\mathcal{T}_{\mathcal{C}}^{(2)}$ to obtain $\mathcal{T}_{\mathcal{C}}$. Hence $\mathcal{T}_{\mathcal{C}}$ represents all embeddings that are represented by both $\mathcal{T}_{\mathcal{C}}^{(1)}$ and $\mathcal{T}_{\mathcal{C}}^{(2)}$. For the converse let $\mathcal{E}_{\mathcal{C}}$ be an embedding represented by $\mathcal{T}_{\mathcal{C}}$. Due to the construction of $\mathcal{T}_{\mathcal{C}}$ it is clear that $\mathcal{E}_{\mathcal{C}}$ is also represented by $\mathcal{T}_{\mathcal{C}}^{(1)}$. For $\mathcal{T}_{\mathcal{C}}^{(2)}$ first observe that the common-face constraints ensure that $\mathcal{E}_{\mathcal{C}}$ adheres to the tree structure of $\mathcal{T}_{\mathcal{C}}^{(2)}$, and hence is represented by its underlying C-tree. To show that the constraints on the crucial relative positions of $\mathcal{T}_{\mathcal{C}}^{(2)}$ are satisfied, we consider such an edge $\left\{C, C^{\prime}\right\}$ in $\mathcal{T}_{\mathcal{C}}^{(2)}$. We use induction over the length of the path $\pi$ from $C$ to $C^{\prime}$ in $\mathcal{T}_{\mathcal{C}}$ and exploit the crucial-position constraints of $\left\{C, C^{\prime}\right\}$ and the common-face constraints on $\pi$.

In the full version of this paper, we show that given a planar graph $G$ and a set of disjoint cycles $\mathcal{C}$ in $C$, a CC-tree of $G$ can be computed in linear time. Moreover, we show that the intersection of two CC-trees can be computed in linear time as well. This immediately yields that Simultaneous Embedding WITH Fixed EDGES can be solved in $O(n)$ time if the common graph consists of a set of disjoint cycles. This result extends to $k$ input graphs, all sharing the same subgraph consisting of disjoint cycles.

Theorem 6. SEFE can be solved in $O(n)$ time for $k$ graphs $G^{(1)}, \ldots, G^{\circledR}$, all intersecting in the same common graph $G$ consisting of disjoint cycles.

\section{Connected Components with Fixed Embedding}

In this section we consider the case where the common graph consists of arbitrary connected components, each with a fixed planar embedding. Again it suffices to care about the relative positions of connected components to one another. Now, if $C$ and $C^{\prime}$ are two connected components in $G$, the relative position $\operatorname{pos}_{C}\left(C^{\prime}\right)$ of $C^{\prime}$ with respect to $C$ is a face of $C$.

We extend the constrained cycle-tree to a constrained component-tree (called $\mathrm{CC}^{\oplus}$-tree) representing all planar embeddings of $G$ that can be induced by an embedding of $G^{(1)}$. As before, each incidence in the $\mathrm{CC}^{\oplus}$-tree represents the decision for a crucial relative position. Moreover, there is a set of inequalities and equations between these choices. As before, $\mathrm{CC}^{\oplus}$-trees can be intersected, again yielding a $\mathrm{CC}^{\oplus}$-tree. To obtain a simultaneous embedding we need to choose relative positions according to the constraints in the $\mathrm{CC}^{\oplus}$-trees. However, since these decisions are not binary anymore, the inequalities yield a coloring problem in the conflict graph, which is NP-hard in general. Due to the special structure of the extended PR-node and cutvertex constraints, inequalities only occur for crucial relative positions that are restricted to at most two values, which again yields a binary choice. We show that the $\mathrm{CC}^{\oplus}$-tree can be computed in $O\left(n^{2}\right)$ time, and that two such trees can be intersected in $O\left(n^{2}\right)$ time.

Theorem 7. SEFE can be solved in $O\left(n^{2}\right)$ time if the embedding of each connected component of the common graph is fixed. 


\section{Conclusion}

Contrasting previous work on the SEFE problem, which makes strong connectivity assumptions on the input graphs, and focus on orderings of edges around vertices, we considered disconnected instances and focused on consistent relative positions. We reduced the general case to instances where the input graphs are connected and gave efficient algorithms for the case where the edge orderings of the common graph are fixed; in particular a linear-time algorithm for instances whose common graph is a set of disjoint cycles. The solutions are based on a compact and easy-to-handle data structure, the $\mathrm{CC}$-tree and $\mathrm{CC}^{\oplus}$-tree, representing all simultaneous embeddings.

\section{References}

1. Angelini, P., Di Battista, G., Frati, F., Jelínek, V., Kratochvíl, J., Patrignani, M., Rutter, I.: Testing planarity of partially embedded graphs. In: Proceedings of the 21st Annual ACM-SIAM Symposium on Discrete Algorithms, SODA 2010, pp. 202-221. SIAM (2010)

2. Angelini, P., Di Battista, G., Frati, F., Patrignani, M., Rutter, I.: Testing the Simultaneous Embeddability of two Graphs whose Intersection is a Biconnected or a Connected Graph. J. Discr. Alg. 14, 150-172 (2012), http://dx.doi.org/10.1016/j.jda.2011.12.015

3. Bläsius, T., Kobourov, S.G., Rutter, I.: Simultaneous embedding of planar graphs. CoRR abs/1204.5853 (2012)

4. Bläsius, T., Rutter, I.: Simultaneous PQ-Ordering with Applications to Constrained Embedding Problems. CoRR abs/1112.0245 (2011)

5. Di Battista, G., Tamassia, R.: On-Line Maintenance of Triconnected Components with SPQR-Trees. Algorithmica 15(4), 302-318 (1996)

6. Di Battista, G., Tamassia, R.: On-Line Planarity Testing. SIAM J. Comput. 25(5), 956-997 (1996)

7. Fowler, J.J., Gutwenger, C., Jünger, M., Mutzel, P., Schulz, M.: An SPQR-Tree Approach to Decide Special Cases of Simultaneous Embedding with Fixed Edges. In: Tollis, I.G., Patrignani, M. (eds.) GD 2008. LNCS, vol. 5417, pp. 157-168. Springer, Heidelberg (2009)

8. Gassner, E., Jünger, M., Percan, M., Schaefer, M., Schulz, M.: Simultaneous Graph Embeddings with Fixed Edges. In: Fomin, F.V. (ed.) WG 2006. LNCS, vol. 4271, pp. 325-335. Springer, Heidelberg (2006)

9. Gutwenger, C., Mutzel, P.: A Linear Time Implementation of SPQR-Trees. In: Marks, J. (ed.) GD 2000. LNCS, vol. 1984, pp. 77-90. Springer, Heidelberg (2001)

10. Haeupler, B., Jampani, K.R., Lubiw, A.: Testing Simultaneous Planarity When the Common Graph Is 2-Connected. In: Cheong, O., Chwa, K.-Y., Park, K. (eds.) ISAAC 2010, Part II. LNCS, vol. 6507, pp. 410-421. Springer, Heidelberg (2010)

11. Jünger, M., Schulz, M.: Intersection Graphs in Simultaneous Embedding with Fixed Edges. Journal of Graph Algorithms and Applications 13(2), 205-218 (2009)

12. Whitney, H.: Congruent Graphs and the Connectivity of Graphs. American Journal of Mathematics 54(1), 150-168 (1932) 